\title{
Prevalência da Síndrome Pré-Menstrual em mulheres em idade reprodutiva
}

\author{
Prevalence of Premenstrual Syndrome in women of reproductive age \\ Prevalencia del Síndrome Premenstrual en mujeres en edad reproductiva
}

Recebido: 12/06/2021 | Revisado: 19/06/2021 | Aceito: 20/06/2021 | Publicado: 05/07/2021

\author{
Larissa Maria Lima \\ ORCID: https://orcid.org/0000-0002-5251-0200 \\ Universidade Federal do Piaú, Brasil \\ E-mail: larissaufpi@hotmail.com \\ Márcia Facundo Romão \\ ORCID: https://orcid.org/0000-0003-0848-7338 \\ Universidade Federal do Piauí, Brasil \\ E-mail: marcinha.romao.mr@gmail.com \\ Guilherme Pertinni de Morais Gouveia \\ ORCID: https://orcid.org/0000-0001-6470-2341 \\ Universidade Federal do Piauí, Brasil \\ E-mail: gpfatufpi@gmail.com
}

\begin{abstract}
Resumo
Objetivo: Verificar a prevalência da SPM em mulheres em idade reprodutiva da cidade de Parnaíba-PI, bem como traçar o perfil clínico e sociodemográfico das mulheres com síndrome pré-menstrual. Método: Tratou-se de um estudo transversal de abordagem quantitativa que contou com uma população de 705 participantes em idade reprodutiva. Os dados da amostra foram coletados por meio de questionários físicos e online com perguntas objetivas. O diagnóstico se deu pelas escalas de SPM e TDPM, baseadas na ACOG e DSM-V, respectivamente. Resultados: O estudo apontou uma prevalência de 99,6\% (IC99\%: 0,001 - 0,014) para mulheres com a SPM, sendo maior parcela desta porcentagem classificada como SPM severa, além de apresentarem distúrbios físicos e emocionais decorrentes da SPM. Conclusão: De acordo com o estudo, recomenda-se realização de estudos longitudinais a fim de mapear e analisar os reais fatores que influenciam no surgimento e persistência da sintomatologia da SPM, bem como medidas preventivas de tais consequências.
\end{abstract}

Palavras-chave: Ciclo menstrual; Tensão pré-menstrual; Transtorno disfórico; Qualidade de vida.

\begin{abstract}
Aim: To verify the prevalence of PMS in women of reproductive age in the city of Parnaíba-PI, as well as to trace the clinical and sociodemographic profile of women with premenstrual syndrome. Method: This was a cross-sectional study with a quantitative approach that included a population of 705 participants of reproductive age. Sample data were collected through physical and online questionnaires with aim questions. Diagnosis was made using the SPM and TDPM scales, based on the ACOG and DSM-V, respectively. Results: The study showed a prevalence of $99.6 \%$ (CI99\%: 0.001 - 0.014) for women with PMS, with a greater portion of this percentage classified as severe PMS, besides having physical and emotional disorders resulting from PMS. Conclusion: According to the study, it is recommended to carry out longitudinal studies in order to map and analyze the real factors that influence the emergence and persistence of PMS symptoms, as well as preventive measures for such consequences.
\end{abstract}

Keywords: Menstrual cycle; Premenstrual tension; Dysphoric disorder; Quality of life.

\section{Resumen}

Objetivo: Verificar la prevalencia de SPM en mujeres en edad reproductiva de la ciudad de Parnaíba-PI, así como trazar el perfil clínico y sociodemográfico de mujeres con síndrome premenstrual. Método: Estudio transversal con abordaje cuantitativo que incluyó una población de 705 participantes en edad reproductiva. Los datos de muestra se recopilaron a través de cuestionarios físicos y en línea con preguntas objetivas. El diagnóstico se realizó mediante las escalas SPM y TDPM, basadas en ACOG y DSM-V, respectivamente. Resultados: El estudio mostró una prevalencia de 99.6\% (IC99\%: 0.001 - 0.014) para mujeres con SPM, con una mayor porción de este porcentaje clasificada como SPM severo, además de tener trastornos físicos y emocionales resultantes del SPM. Conclusión: Según el estudio, se recomienda realizar estudios longitudinales con el fin de mapear y analizar los factores reales que influyen en la aparición y persistencia de los síntomas del SPM, así como las medidas preventivas para tales consecuencias.

Palabras clave: Ciclo menstrual; Tensión premenstrual; Trastorno disfórico; Calidad de vida. 


\section{Introdução}

O ciclo menstrual é caracterizado por alterações fisiológicas ocorridas nas mulheres em idade reprodutiva, é regulado pelo sistema neuroendócrino e está subdividido em 3 fases: folicular - na qual ocorre a produção do hormônio folículoestimulante (FSH), levando os ovários a amadurecer seus óvulos; ovulatória - marcada pela contínua elevação de estrogênio e produção do hormônio luteinizante (LH), responsável pela escolha do óvulo maduro e expulsão do mesmo; e pela fase lútea responsável por produzir progesterona em maior quantidade, preparando o útero para uma possível gravidez (Geleski et al., 2018). Uma resposta neural mal adaptada poderá produzir transtornos relacionados aos hormônios deste ciclo, como o que acontece na Síndrome Pré-Menstrual - SPM (Paiva et al., 2010).

Podemos retratar a SPM como a soma de sinais e sintomas comportamentais, emocionais e físicos vivenciados pela mulher uma semana antes da menstruação, na etapa conhecida como fase lútea, e que tende a desaparecer com o início da menstruação (Macambira \& Golino, 2018). Este conjunto de sinais e sintomas variam em quantidade e intensidade entre as mulheres. Sintomas leves que não influenciam na rotina não são considerados como diagnóstico de SPM, pois para se caracterizar como síndrome é necessário que interfira negativamente nas atividades de vida diária (Silva et al., 2020). Dentre os sintomas físicos mais comuns estão edema abdominal, edema nas mamas e nas extremidades, dores musculares e articulares, fadiga, dor de cabeça, aumento do apetite e ganho de peso. Já entre os sintomas psíquicos, destacam-se raiva, ansiedade, depressão, humor variável, dificuldade de concentração, nervosismo e sensação de desespero, levando a padrões de comportamento alterado, principalmente na fase lútea tardia da menstruação (Yilmaz-Akyuz \& Aydin-Kartal, 2019).

Atualmente, a SPM afeta milhares de mulheres no mundo todo, sendo o maior distúrbio que acomete mulheres em fase reprodutiva, e também o mais comum (Maia et al., 2014). Epidemiologicamente, $75 \%$ a 95\% das mulheres em idade fértil sofrem de algum sintoma referente à Síndrome da Tensão Pré-Menstrual (STPM) (Santos et al., 2020). O grau de disfunção irá variar de acordo com a intensidade em que a síndrome se apresenta, podendo apresentar sintomas variados e inconstantes, interferindo levemente ou significativamente na qualidade de vida da mulher (Alves et al., 2019).

Existem diversas modalidades que podem atuar sobre tal síndrome, como por exemplo a fisioterapia uroginecológica, que conta com técnicas e protocolos que visam a melhora de doenças e disfunções. A procura por essas alternativas terapêuticas eficientes e seguras, capazes de atuar nos sintomas psicológicos e físicos podem trazer benefícios socioeconômicos e qualidade de vida para as mulheres que sofrem com esse grande transtorno (Santos et al., 2020).

Considerando-se que a sintomatologia altera de forma significativa a qualidade de vida dessas mulheres, tendo também uma escassez de artigos relacionados ao tema e abordando a fisioterapia na minimização dos sintomas da SPM, bem como a necessidade de encontrar métodos eficazes para a redução de sintomas da SPM, procura-se a partir desse estudo verificar a prevalência da SPM em mulheres em idade reprodutiva da cidade de Parnaíba-PI, bem como traçar o perfil clínico e sociodemográfico das mulheres com síndrome pré-menstrual, para que seja possível, futuramente, oferecer cada vez mais alternativas terapêuticas, trazendo à tona esse assunto pouco abordado e que atinge tantas mulheres.

\section{Metodologia}

Esse trabalho tratou-se de um estudo transversal de abordagem quantitativa, realizado no Grupo de Pesquisa em Fisioterapia Avaliativa e Terapêutica - GPFAT, na Universidade Federal do Piauí no período de setembro de 2019 a dezembro de 2020. Segundo Hochman et al. (2005), estudos do tipo transversal são utilizados para detecção de casos por meio do cálculo da proporção entre pessoas acometidas e pessoas saudáveis, ideal para estudos de prevalência. Os dados coletados foram analisados no final da coleta por meio de técnicas matemáticas, conforme Pereira et al. (2018).

Participaram desta pesquisa 705 mulheres em idade reprodutiva, entre 18 e 30 anos, que residiam na cidade de Parnaíba-PI e regiões adjacentes e que consentiram participar mediante a leitura e assinatura do Termo de Consentimento Livre 
e Esclarecido - TCLE. Foram excluídas mulheres que não menstruaram nos 3 últimos meses que antecederam o preenchimento do questionário ou que o tivessem preenchido de forma incompleta impossibilitando o diagnóstico, mulheres que residiam fora do local definido para estudo e aquelas que retiraram o seu consentimento.

Para cálculo amostral, utilizou-se o software GPower®, post hoc, devido o cálculo inicial não ter sido atingido por conta da pandemia, obtendo 705 voluntárias [Power $(1-\beta$ err prob $=92,9 \%) ; \alpha$ err prob $=0,05$; effect size $=5 \%$; constante de porporção $=0,2]$.

Esta pesquisa foi cadastrada e aprovada pelo Comitê de Ética em Pesquisa da Universidade Federal do Piauí - CEP UFPI sob parecer número 3.943.247. O estudo foi realizado seguindo princípios éticos da Resolução 466/12 do Conselho Nacional de Saúde - CNS (2012), respeitando os quatro referenciais básicos da bioética: autonomia, não maleficência, beneficência e justiça. Os pesquisadores também se disponibilizaram a esclarecer quaisquer dúvidas sobre os questionários, reiterando que haveria sigilo das informações oferecidas e anonimato, sem qualquer risco ou prejuízo as suas atividades; que a participação não acarretaria ônus nem bônus às participantes, e que as mesmas teriam direito a se retirar do estudo se assim desejassem, em qualquer etapa e sem prejuízos.

A coleta de dados se deu por meio da aplicação do questionário (físico ou online) com perguntas objetivas elaborado pelos pesquisadores Muramatsu et al. (2001), do qual após ser adaptado ao estudo, foi retirada a parte A, em vista de ter sido substituída pelo questionário sociodemográfico, e utilizada somente a parte $\mathrm{B}$, que apresentava uma gama de sintomas físicos e emocionais, seguidos de questões envolvendo as possíveis consequências de tais sintomas no dia a dia. O diagnóstico foi obtido de acordo com o American College of Obstetricians and Gynecologists - ACOG (2009) que caracteriza como SPM com sintomatologia leve, tendo até três sintomas físicos ou emocionais, e moderada quando se relata até quatro sintomas. Devido a instabilidade dos sintomas de ciclo a ciclo, é necessário que esses estejam presentes em pelo menos de dois a três ciclos consecutivos.

Além deste, questionou-se também sobre o Transtorno Disfórico Pré-Menstrual (TDPM) de acordo com os critérios diagnósticos estabelecidos no Manual Diagnóstico e Estatístico de Transtornos Mentais - 5a Edição (DSM-5) (2014), o qual determina como TDPM a presença de pelo menos cinco ou mais sintomas na semana final antes do início da menstruação, com no mínimo um deles pertencendo a um dos quatro primeiros citados no DSM-5; e melhora do quadro poucos dias após o início da menstruação, tornando-se mínimos ou ausentes na semana pós-menstrual. O TDPM é o subtipo da SPM caracterizado como grave. Seu diagnóstico clínico pode determinar um tratamento mais satisfatório das mulheres com a SPM (Arruda et al., 2011).

Os questionários eram compostos por questões objetivas, cujas respostas deveriam ser marcadas de acordo com as implicações no dia a dia da mulher entrevistada e cujo preenchimento levava em torno de cinco a oito minutos. Os dados coletados por meio dos questionários físicos e online foram tabulados no programa de edição de planilhas, Microsoft Excel ${ }^{\circledR}$. Os questionários on-line foram desenvolvidos no aplicativo Google Forms.

Para realização da estatística, utilizou-se análise descritiva dos dados com valores absolutos e percentual, médias $( \pm$ Dp), além de Intervalo de Confiança (IC99\% e IC95\%), teste Qui-quadrado de Wald, razão de prevalência bruta e ajustada, bem como o método de regressão de Poisson, por ser direcionado a altas prevalências. Considerou-se como significância estatística quando o valor $p$ fosse menor ou igual a 0,05 .

\section{Resultados}

O estudo contemplou 705 voluntárias, apesar do número expressivo, este ficou aquém do cálculo amostral devido ao período de pandemia e medidas de lockdown, interferindo nas aulas presenciais e, diretamente, na coleta dos dados. 
Mesmo os questionários apresentando lacunas vazias (sem respostas), optou-se por manter a todas as respostas, uma vez que a ausência de respostas foi inferior a 3,0\%. Por este motivo, alguns dados nas tabelas apresentarão valores de $n$ diferentes.

A média de idade das participantes foi 21,78 anos (18 - 30; $\pm 2,9$ anos). Destas, $54,4 \%$ autodeclararam pardas e $87,2 \%$ relataram estar solteiras no momento do preenchimento do questionário. A renda familiar da população em estudo apresentou mediana de 1.500,00 reais e média de 2.203,00 ( \pm 2.510,17 reais), estando classificada em Classe E (63,9\%). Em relação à ocupação, 76,5\% é apenas estudante, quanto as demais, exercem uma outra profissão como fisioterapeuta, professora, dentre outras. A Tabela 1 mostra que 94,7\% das mulheres residem em Parnaíba - PI, cursando Fisioterapia (22,8\%), 45,1\% estando até a metade do curso. Quanto à orientação sexual, 82,1\% das mulheres se declararam heterossexual, sendo 62,0\% das entrevistadas com vida sexual ativa. Já em relação à menstruação, 70,4\% das voluntárias relataram apresentar regularidade do ciclo, ocorrendo, em média, a cada 29,28 dias com mediana de 28 dias $(15-180 ; \pm 8,87$ dias), tendo média de 5,49 dias por ciclos $(3-15 ; \pm 3,82$ dias $)$.

Tabela 1: Características socioeconômicas, demográficas e de comportamento das voluntárias do estudo. Parnaíba, PI, 2021.

\begin{tabular}{|c|c|c|}
\hline Variáveis & $\mathbf{N}$ & $\%$ \\
\hline \multicolumn{3}{|c|}{ Faixa etária (anos)* } \\
\hline $18-19$ & 163 & 23,3 \\
\hline $20-24$ & 417 & 59,4 \\
\hline $25-30$ & 121 & 17,3 \\
\hline \multicolumn{3}{|c|}{ Cor da pele / etnia* } \\
\hline Branca & 220 & 31,3 \\
\hline Parda & 382 & 54,4 \\
\hline Negra & 97 & 13,8 \\
\hline Não declarada & 3 & 0,5 \\
\hline \multicolumn{3}{|l|}{ Estado Civil* } \\
\hline Solteira & 598 & 87,2 \\
\hline Casada & 33 & 4,8 \\
\hline Divorciada & 3 & 0,4 \\
\hline Outro & 52 & 7,6 \\
\hline \multicolumn{3}{|l|}{ Residência* } \\
\hline Parnaíba & 648 & 94,7 \\
\hline Luís Correia & 14 & 2,0 \\
\hline Buriti dos Lopes & 12 & 1,8 \\
\hline Cajueiro da Praia & 3 & 0,4 \\
\hline Ilha Grande & 7 & 1,0 \\
\hline \multicolumn{3}{|l|}{ Ocupação* } \\
\hline Estudante & 525 & 76,5 \\
\hline Fisioterapeuta & 25 & 3,6 \\
\hline Professora & 21 & 3,1 \\
\hline Psicóloga & 11 & 1,6 \\
\hline Desempregada & 10 & 1,5 \\
\hline Outra & 94 & 13,7 \\
\hline
\end{tabular}




\section{Renda familiar em SM*}

\begin{tabular}{|c|c|c|}
\hline Inferior a $1 \mathrm{SM}$ & 156 & 23,2 \\
\hline 1 a $2 \mathrm{SM}$ & 273 & 40,7 \\
\hline 2 a 4 SM & 157 & 23,4 \\
\hline 4 a $10 \mathrm{SM}$ & 67 & 10,1 \\
\hline 10 a $15 \mathrm{SM}$ & 17 & 2,5 \\
\hline Acima de $20 \mathrm{SM}$ & 1 & 0,1 \\
\hline \multicolumn{3}{|l|}{ Curso* } \\
\hline Fisioterapia & 156 & 22,8 \\
\hline Psicologia & 71 & 10,4 \\
\hline Biologia & 66 & 9,6 \\
\hline Biomedicina & 55 & 8,0 \\
\hline Turismo & 45 & 6,6 \\
\hline Pedagogia & 41 & 6,0 \\
\hline Engenharia de Pesca & 25 & 3,6 \\
\hline Enfermagem & 23 & 3,4 \\
\hline Direito & 21 & 3,1 \\
\hline Matemática & 21 & 3,1 \\
\hline Outros & 161 & 23,4 \\
\hline \multicolumn{3}{|l|}{ Orientação Sexual* } \\
\hline Heterossexual & 578 & 82,1 \\
\hline Bissexual & 83 & 11,8 \\
\hline Homossexual & 31 & 4,4 \\
\hline Assexual & 8 & 1,1 \\
\hline Não soube informar & 4 & 0,6 \\
\hline \multicolumn{3}{|l|}{ Vida Sexual* } \\
\hline Ativa & 433 & 62,0 \\
\hline Inativa & 104 & 14,9 \\
\hline Não iniciada & 161 & 23,1 \\
\hline \multicolumn{3}{|c|}{ Regularidade Menstrual } \\
\hline Regular & 496 & 70,4 \\
\hline Irregular & 209 & 29,6 \\
\hline Total & 705 & 100 \\
\hline
\end{tabular}

Legenda: N - número de voluntárias para cada observação (categoria); SM - salário mínimo; *Variáveis que apresentaram até $3,0 \%$ de respostas ignoradas. Fonte: Lima; Romão; Gouveia (2021).

A Figura 1 apresenta a prevalência de cada sintoma pré-menstrual de acordo com as categorias analisadas: físico e emocional. Em relação aos sintomas físicos (Figura 1A), os mais frequentes foram: mastalgia, acne, cólicas, desejo por certos tipos de alimentos e dor nas costas, todos acima de 50\% de frequência. Já os sintomas emocionais (Figura 1B) mais prevalentes foram: impaciência, vontade de chorar, distimia, ansiedade, tristeza e raiva, todos com frequência superior a 55\%. Percebe-se que a Síndrome Pré-Menstrual gera uma grande influência, principalmente, nos sintomas emocionais. Quando usados o critério DSM-V modificado e a classificação pela American College of Obstetricians and Gynecologists - ACOG 
(2009), observou-se uma prevalência de 99,6\% (IC99\%: 0,001 - 0,014) para mulheres com a Síndrome Pré-menstrual, sendo categorizada em leve $(9,5 \%)$, moderada $(33,0 \%)$ e severa $(57,5 \%)$.

Figura 1: Prevalência e distribuição dos sintomas pré-menstruais das voluntárias em estudo (N = 705). Parnaíba, PI, 2021.

\section{A (Sintomas Físicos)}

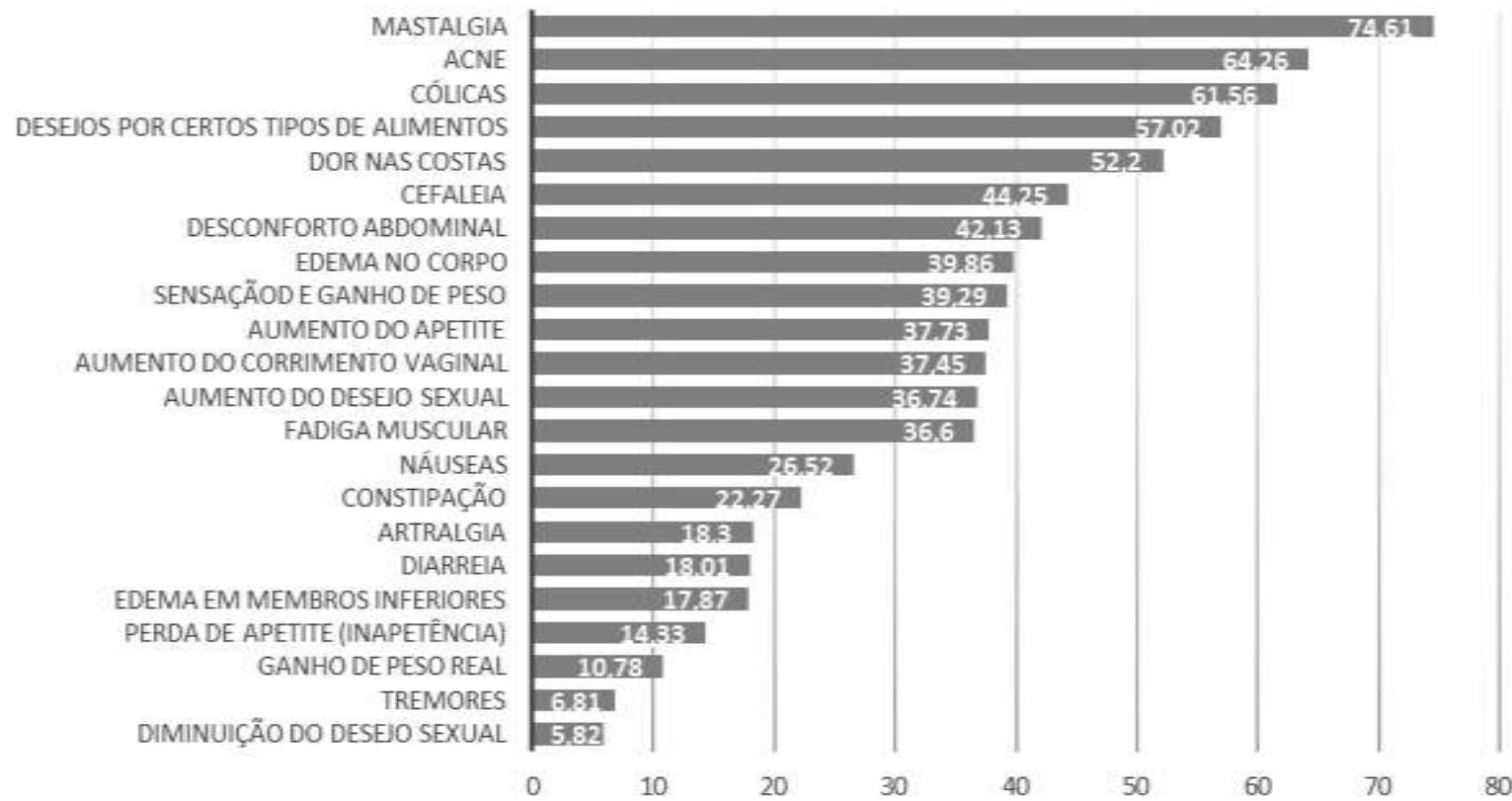

\section{B (Sintomas Emocionais)}

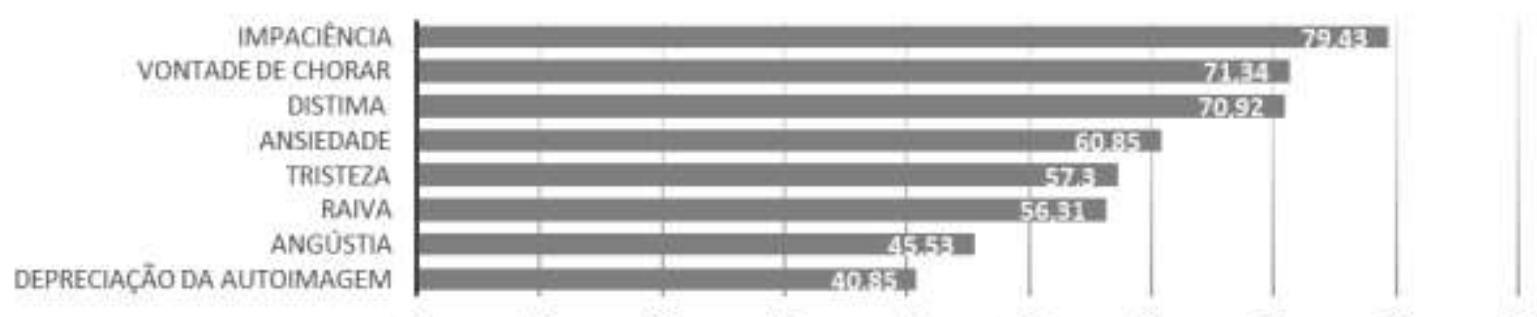

Legenda: A: sintomas físicos em porcentagem; B: Sintomas emocionais em porcentagem. Fonte: Lima; Romão; Gouveia (2021).

De modo geral, ao analisar as repercussões ocasionadas pela exacerbação dos sintomas físicos e psicológicos que acarretam em consequências sócio-ocupacional, familiar e conjugal, observou-se predominância nos quesitos: respostas hostis às pessoas $(48,79 \%)$, interesse diminuído pelo estudo/trabalho $(48,65 \%)$, aumento das brigas com filhos $(47,61 \%)$, os filhos dão mais trabalhos $(26,19 \%)$, briga com os familiares mesmo sem motivos $(65,81 \%)$ e percebem que o cônjuge briga mais com elas $(20,59 \%)$, conforme apresentado na Tabela 2. 
Tabela 2: Prevalência das consequências da SPM no comportamento sócio-ocupacional, familiar e conjugal das voluntárias do estudo. Parnaíba, PI, 2021.

\begin{tabular}{|c|c|c|}
\hline Variáveis & $\mathbf{N}$ & $\%$ \\
\hline \multicolumn{3}{|l|}{ Consequências no trabalho/faculdade } \\
\hline Respostas hostis às pessoas & 344 & 48,79 \\
\hline Interesse diminuído pelo estudo/trabalho & 343 & 48,65 \\
\hline Dificuldade de concentração e de manter a atenção fixa & 341 & 48,36 \\
\hline Sonolência & 289 & 40,99 \\
\hline Distração & 280 & 39,71 \\
\hline Desorganização no estudo e no trabalho & 266 & 37,73 \\
\hline Não consegue terminar suas tarefas & 204 & 28,93 \\
\hline Quietude & 175 & 24,82 \\
\hline Insônia & 161 & 22,83 \\
\hline Gera clima de tensão & 146 & 20,70 \\
\hline Comete mais erros & 125 & 17,73 \\
\hline Absenteísmo & 90 & 12,76 \\
\hline Chega atrasada & 85 & 12,05 \\
\hline Acidenta-se facilmente & 74 & 10,49 \\
\hline \multicolumn{3}{|l|}{ Relacionamento com filhos* } \\
\hline Briga mais com eles & 20 & 47,61 \\
\hline Não relatou alterações & 14 & 33,3 \\
\hline Sai/passeia mais com os filhos & 4 & 9,52 \\
\hline Ofende-os verbalmente & 3 & 7,14 \\
\hline Ignora-os & 2 & 4,76 \\
\hline Procura deixar os filhos com a avó ou outra pessoa & 1 & 2,38 \\
\hline Procura não brigar com eles & 1 & 2,38 \\
\hline \multicolumn{3}{|l|}{ Reações dos filhos* } \\
\hline Não apresentam reações negativas & 19 & 45,23 \\
\hline Dão mais trabalho & 11 & 26,19 \\
\hline Choram mais & 7 & 16,67 \\
\hline Carência & 6 & 14,28 \\
\hline Medo/desobediência ou rebeldia & 5 & 11,90 \\
\hline Irritabilidade & 4 & 9,52 \\
\hline \multicolumn{3}{|l|}{ Relacionamento com familiares } \\
\hline Briga com eles, mesmo sem motivos & 464 & 65,81 \\
\hline Ignora-os & 195 & 27,65 \\
\hline Isola-os & 158 & 22,41 \\
\hline Cria intrigas com e entre eles & 109 & 15,46 \\
\hline Não apresentou alterações & 96 & 13,61 \\
\hline Ofende-os verbalmente & 86 & 12,19 \\
\hline \multicolumn{3}{|l|}{ Mudanças no comportamento do esposo(a) ou namorado(a)** } \\
\hline Não percebe mudanças & 175 & 52,23 \\
\hline Discute mais com você & 69 & 20,59 \\
\hline
\end{tabular}

Legenda: SPM: Síndrome Pré-Menstrual; N - número de voluntárias para cada observação (categoria); *N= 42, referente ao quantitativo de mães. ${ }^{* *} \mathrm{~N}=335$, referente àquelas que tem esposo(a) ou namorado(a). Fonte: Lima; Romão; Gouveia (2021). 
No que se refere ao Transtorno Disfórico Pré-Menstrual (TDPM), observa-se que as mais prevalentes, de acordo com a Figura 2, são: ansiedade acentuada, tensão, nervos à flor da pele (66,16\%); raiva ou irritabilidade persistente e conflitos interpessoais (60,35\%); cansaço excessivo com diminuição da capacidade mental, fadiga fácil ou acentuada falta de energia (57,07\%); outros sintomas físicos como sensibilidade ou inchaço nas mamas, dor de cabeça, dor articular ou muscular, sensação de inchaço geral e ganho de peso $(54,24 \%)$ e sente-se deprimida, com sentimentos de falta de esperança ou autodepreciativos $(52,93 \%)$.

Figura 2: Prevalência e distribuição dos itens avaliados no Transtorno Disfórico Pré-Menstrual (TDPM) das voluntárias em estudo que apresentaram estas características $(\mathrm{N}=671)$. Parnaíba, PI, 2021.

\section{TDPM}

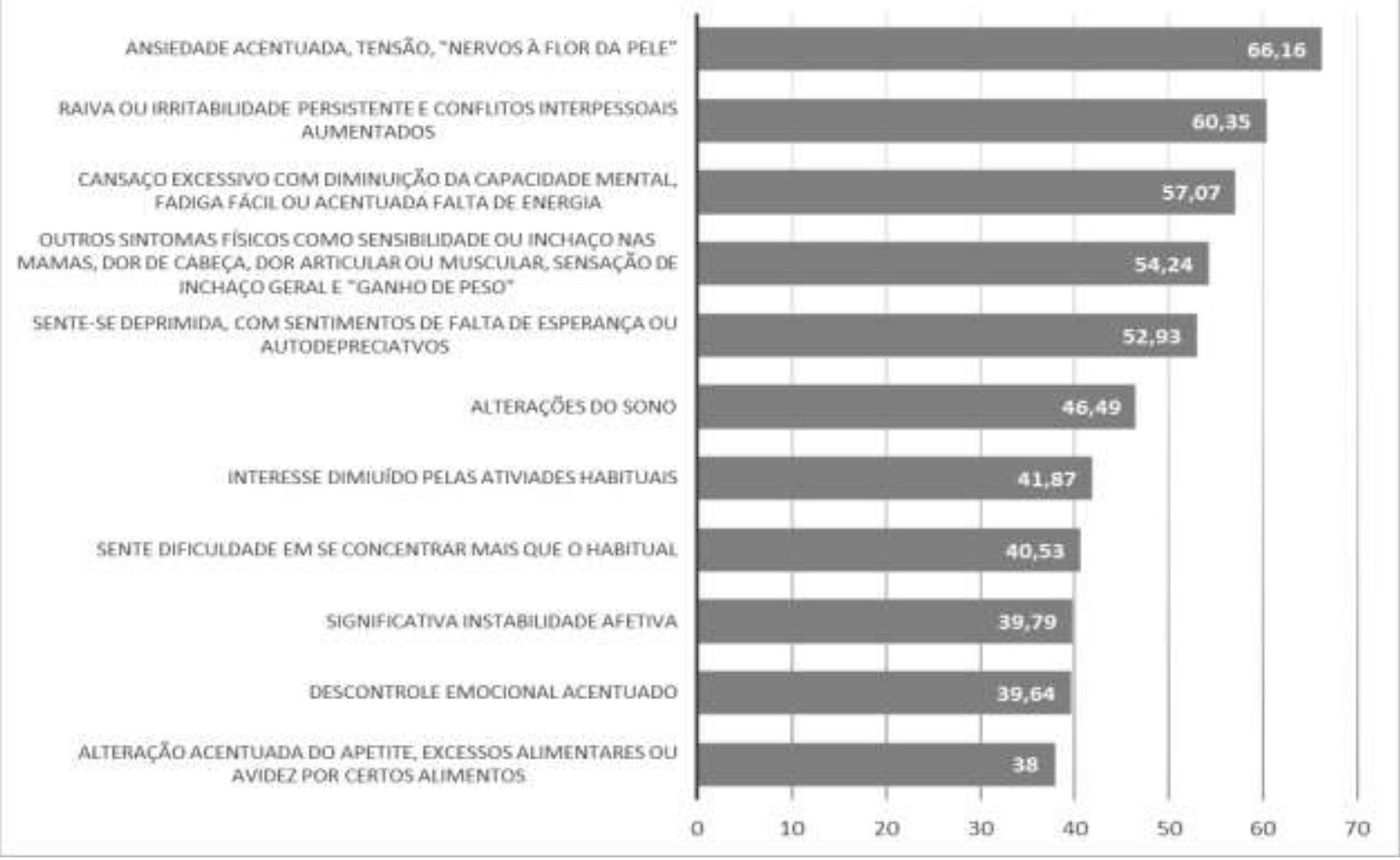

Legenda: TDPM: Transtorno Disfórico Pré-Menstrual em porcentagem. Fonte: Lima; Romão; Gouveia (2021).

Para análise de regressão de Poisson, considerou-se a gravidade da SPM por meio do TDPM, tendo como desfecho principal a classificação severa. Desta forma, a Tabela 3 mostra que a SPM severa é mais prevalente em mulheres com idade variando de 20 a 24 anos $(59,9 \%)$, parda $(54,1 \%)$, vida sexual ativa $(61,6 \%)$, cursando a graduação $(77,4 \%)$ e com renda familiar variando entre 1 e 2 salários mínimos $(41,0 \%)$. 
Tabela 3: Prevalência de Síndrome Pré-menstrual severa por variáveis socioeconômicas, demográficas e de comportamento para mulheres em idade fértil (N=705). Parnaíba, PI, 2021.

\begin{tabular}{|c|c|c|}
\hline Variáveis & \multicolumn{2}{|l|}{ SPM } \\
\hline Faixa etária (anos) & & $0,026^{\phi}$ \\
\hline 18 e 19 & 24,1 & \\
\hline 20 a 24 & 59,9 & \\
\hline 25 a 30 & 16,0 & \\
\hline Cor da pele & & $0,035^{\phi}$ \\
\hline Branca & 31,9 & \\
\hline Parda & 54,1 & \\
\hline Negra & 13,9 & \\
\hline Não declarada & 0,1 & \\
\hline Estado Civil & & 0,838 \\
\hline Solteira & 87,1 & \\
\hline Casada & 4,4 & \\
\hline Divorciada & 0,4 & \\
\hline Outro & 8,1 & \\
\hline Orientação sexual & & 0,414 \\
\hline Homossexual & 4,2 & \\
\hline Heterossexual & 82,4 & \\
\hline Bissexual & 11,4 & \\
\hline Assexual & 1,3 & \\
\hline Indefinido / não soube opinar & 0,7 & \\
\hline Vida sexual & & $0,001^{\phi}$ \\
\hline Ativa & 61,6 & \\
\hline Inativa & 15,3 & \\
\hline Não iniciada & 23,1 & \\
\hline Grau de escolaridade & & $<0,0001^{\phi}$ \\
\hline Ensino fundamental completo & 0,5 & \\
\hline Ensino médio & 6,1 & \\
\hline Ensino superior incompleto (cursando a graduação) & 77,4 & \\
\hline Ensino superior completo & 14,7 & \\
\hline Especialização & 1,0 & \\
\hline Mestrado & 0,3 & \\
\hline Renda familiar (faixa) & & $0,041^{\phi}$ \\
\hline Inferior a $1 \mathrm{SM}$ & 23,0 & \\
\hline 1 a $2 \mathrm{SM}$ & 41,0 & \\
\hline 2 a 4 SM & 23,0 & \\
\hline 4 a 10 SM & 10,4 & \\
\hline 10 a $15 \mathrm{SM}$ & 2,4 & \\
\hline Acima de 20 SM & 0,2 & \\
\hline Regularidade menstrual & & 0,804 \\
\hline Regular & 72,8 & \\
\hline Irregular & 27,2 & \\
\hline
\end{tabular}

Legenda: SPM: Síndrome Pré-Menstrual; SM - salário Mínimo; *Teste Qui-quadrado de Wald; \$ Significância estatística. Fonte: Lima; Romão; Gouveia (2021).

Na Tabela 4, após análise ajustada, permaneceram associadas à SPM severa: cor da pele (parda e negra), vida sexual, renda familiar e regularidade menstrual. O risco da SPM severa foi 60,4\% e 61,9\% maior em mulheres pardas e negras, respectivamente, que tinham maior renda familiar $(72 \%)$ e vida sexual ativa (83\%). A regularidade menstrual mostrou-se protetora da SPM severa. Todavia, o tempo do ciclo menstrual não apresentou influência significativa no aparecimento da SPM severa [1,003 $(0,9-1,006), p=0,091]$, entretanto, os dias de duração da menstruação mostrou-se significativo [1,086 $(1,042-1,131), p<0,0001]$. 
Tabela 4: Razões de prevalências brutas e ajustadas para Síndrome Pré-menstrual Severa, de acordo com as variáveis socioeconômicas, demográficas e de comportamento para mulheres em idade fértil (N=705). Parnaíba, PI, 2021.

\begin{tabular}{|c|c|c|}
\hline Variável & $\begin{array}{l}\text { SPM bruta } \\
\text { RP }(\text { IC 95\%)* }\end{array}$ & $\begin{array}{l}\text { SPM ajustada } \\
\text { RP }(\text { IC 95\%)** }\end{array}$ \\
\hline Faixa etária (anos) & $0,05 * * *$ & $0,026^{* * *}$ \\
\hline 18 e 19 & $2,396(0,884-6,490)$ & $2,434(0,881-6,726)$ \\
\hline 20 a 24 & $2,386(0,89-6,397)$ & $2,447(0,893-6,706)$ \\
\hline 25 a 30 & 1 & 1 \\
\hline Cor da pele & $0,035 * * *$ & $0,033 * * *$ \\
\hline Branca & $0,612(0,404-0,927)$ & $0,653(0,426-1,001)$ \\
\hline Parda & $0,559(0,372-0,841)$ & $0,604(0,397-0,921)$ \\
\hline Negra & $0,566(0,364-0,881)$ & $0,619(0,390-0,981)$ \\
\hline Não declarada & 1 & 1 \\
\hline Estado Civil & $0,838 * * *$ & $0,218 * * *$ \\
\hline Solteira & $0,957(0,768-1,192)$ & $0,948(0,764-1,177)$ \\
\hline Casada & $0,840(0,565-1,247)$ & $0,825(0,549-1,239)$ \\
\hline Divorciada & $0,785(0,215-2,863)$ & $0,717(0,195-2,643)$ \\
\hline Outro & 1 & 1 \\
\hline Orientação sexual & $0,414 * * *$ & $0,263 * * *$ \\
\hline Homossexual & $1,385(0,535-3,584)$ & $1,323(0,48-3,647)$ \\
\hline Heterossexual & $1,311(0,516-3,327)$ & $1,3(0,479-3,526)$ \\
\hline Bissexual & $1,535(0,6-3,924)$ & $1,526(0,559-4,163)$ \\
\hline Assexual & $1,488(0,530-4,172)$ & $1,380(0,456-4,182)$ \\
\hline Indefinido / não soube opinar & 1 & 1 \\
\hline Vida sexual & $0,001 * * *$ & $0,003 * * *$ \\
\hline Ativa & $18,34(14,87-22,61)$ & $18,3(14,91-22,47)$ \\
\hline Inativa & $17,37(13,45-22,43)$ & $17,24(13,41-22,17)$ \\
\hline Não iniciada & 1 & 1 \\
\hline Grau de escolaridade & $<0,0001 * * *$ & $0,539 * * *$ \\
\hline Ensino fundamental completo & $1,821(1,228-2,701)$ & $1,308(0,932-1,838)$ \\
\hline Ensino médio & $0,718(0,499-1,033)$ & $0,785(0,54-1,141)$ \\
\hline $\begin{array}{c}\text { Ensino superior } \\
\text { (cursando a graduação) }\end{array}$ & $0,762(0,626-0,929)$ & $0,835(0,677-1,031)$ \\
\hline Ensino superior completo & $0,699(0,537-0,912)$ & $0,781(0,591-1,031)$ \\
\hline Especialização & $0,702(0,422-1,168)$ & $0,735(0,453-1,193)$ \\
\hline Mestrado & 1 & 1 \\
\hline Renda familiar (faixa) & $0,432 * * *$ & $0,041 * * *$ \\
\hline Inferior a $1 \mathrm{SM}$ & $1,345(0,48-3,520)$ & $1,1(0,00-0,8)$ \\
\hline 1 a $2 \mathrm{SM}$ & $1,312(0,79-3,6)$ & $1,43(0,00-0,91)$ \\
\hline 2 a 4 SM & $1,26(0,59-4,63)$ & $1,36(0,00-0,56)$ \\
\hline 4 a $10 \mathrm{SM}$ & $1,8(0,46-4,82)$ & $1,72(0,00-0,89)$ \\
\hline 10 a $15 \mathrm{SM}$ & $0,995(0,394-2,509)$ & $1,24(0,00-0,506)$ \\
\hline Acima de 20 SM & 1 & 1 \\
\hline Regularidade menstrual & $0,804 * * *$ & $0,043 * * *$ \\
\hline Regular & $1,247(0,74-1,44)$ & $1,44(1,25-1,67)$ \\
\hline Irregular & 1 & 1 \\
\hline
\end{tabular}

Legenda: SPM: Síndrome Pré-Menstrual; SM - salário Mínimo; RP: Razão de Prevalência; *Razão de prevalência bruta e intervalo de confiança de 95\%; ** Razão de prevalência ajustada e intervalo de confiança de 95\%; Teste Qui-quadrado de Wald. Fonte: Lima; Romão; Gouveia (2021).

\section{Discussão}

Corroborando com os dados apresentados neste estudo, Jarosz, Jamnik e El-Sohemy (2017) estudaram 1102 mulheres canadenses multiétnicas e encontraram uma alta prevalência de 99\%, que não diferiu entre os grupos étnicos. Outra pesquisa transversal realizada por Abeje \& Berhanu (2019), em uma escola secundária na Etiópia, com 492 alunas do $9^{\circ}$ ao $12^{\circ}$ ano, verificou uma prevalência de 81,7\% para SPM, segundo os critérios da ACOG (2009). De forma similar, Hardy e Hunter 
(2021) buscaram entender os sintomas pré-menstruais e suas consequências no trabalho, por meio de um questionário online, no qual 125 mulheres participaram. Os autores relataram que a prevalência dos sintomas pré-menstruais foi superior a $90 \%$ nas últimas quatro semanas, sendo que destes, 60,8\% tinham sintomas leves, cerca de 34,4\% apresentou sintomatologia moderada e apenas 4,8\% sintomas graves consideradas com TDPM. Shrestha et al (2019) realizaram um estudo prospectivo, entre 285 estudantes de um hospital universitário no Nepal e observaram que 72,3\% relataram pelo menos um sintoma de SPM de intensidade moderada a grave, todas as estudantes estavam no ensino superior, em cursos da saúde, 92,6\% eram de áreas urbanas e $80,7 \%$ tinham ciclos menstruais regulares, semelhante às características da amostra deste estudo.

Tolossa e Bekele (2014) acreditam que uma alta prevalência entre universitárias se deve possivelmente ao estresse acadêmico, principalmente entre os cursos da saúde. A idade também mostrou-se significante, de acordo com o estudo de Jarosz, Jamnik e El-Sohemy (2017), com quatro grupos étnicos diferentes e com média de idade de 22,6 anos, foi observada uma prevalência de 99\%, que não diferiu entre os grupos. Outro estudo, com 492 mulheres, que também mostrou uma prevalência alta de 81,3\% para SPM, tinha média de idade de 17,61 anos (Abeje \& Berhanu, 2019). Victor et al. (2019) relataram que diferentes características sociodemográficas e de estilo de vida podem influenciar na síndrome, tais como cor da pele, vida sexual, renda familiar e regularidade menstrual no grau de severidade, porém os autores discordam quanto a significância da idade na interferência da SPM leve e moderada.

Divergindo dos resultados encontrados, Yilmaz-Akyuz e Aydin-Kartal (2019) realizaram um estudo em Istambul, na Turquia, contendo 426 participantes que foram monitoradas durante dois ciclos menstruais pela Escala de Síndrome PréMenstrual, observando uma prevalência de 43,8\% para SPM. Os autores ainda relatam que a prevalência pode variar até de 39,86 a 98,1\% a depender do país, cultura e etnias estudadas. Conforme Victor et al. (2019), o fato de os estudos serem feitos com diferentes populações pode interferir no diagnóstico de TDPM, no sentido de apresentar outros diagnósticos diferenciais similares, como transtorno depressivo e de ansiedade, síndrome do intestino irritável e doenças da tireoide, além dos diferentes critérios e escalas utilizados no diagnóstico.

Os sintomas físicos mais frequentemente relatados na SPM e citados neste artigo estavam de acordo com outros trabalhos. 91,2\% das mulheres com essa síndrome relataram sensibilidade mamária, dores de cabeça ou dores articulares e musculares, distensão abdominal, aumento de peso e 84,5\% relataram fadiga ou falta de energia (Hardy \& Hunter, 2021). Outros autores mostraram uma prevalência de 73,4\% para edema abdominal, 25,0\% edema nas extremidades, 23,0\% para cefaleia, 4,7\% relataram seios sensíveis (Abeje \& Berhanu, 2019). Em um estudo com mais de 42 mil mulheres acerca dos sintomas pré-menstruais e seus impactos no dia-a-dia, Schoep et al. (2019) afirmaram que dores nas costas, na cabeça e sangramento menstrual intenso parecem estar presentes em uma a cada duas mulheres, e que $40 \%$ das entrevistadas relataram um desempenho inferior em suas atividades durante a menstruação. A diferença nos valores pode se relacionar a problemas de viés de memória, uma vez que se trata de estudos transversais, que utilizam lembranças retrospectivas sobre os sintomas (Victor et al., 2019).

Os sintomas emocionais como: raiva ou irritabilidade (16,1 a 93,9\%), isolamento social (44,9\%), depressão (43,3\%), confusão $(34,1 \%)$ e sintomas de ansiedade foram os mais prevalentes, corroborando com os resultados deste estudo (Abeje \& Berhanu, 2019; Hardy \& Hunter, 2021); além de ânsia de choro, labilidade emocional e humor deprimido, que segundo Shrestha et al. (2019) foram sintomas relatados por pelo menos $44,2 \%$ das 285 voluntárias pesquisadas. No âmbito do TDPM, Śliwerski e Bielawska-Batorowicz (2019) afirmaram que, cerca de 30 a $80 \%$ das mulheres que sofrem com essa forma mais grave da SPM, já tiveram pelo menos um episódio depressivo no passado. Em um estudo com 3.791 mulheres, Jang e Elfebein (2019) observam que, mulheres com TDPM estavam 17\% mais inclinadas a tentar o suicídio e pelo menos $20 \%$ já foram admitidas em internações psiquiátricas durante o período menstrual, quando comparadas a mulheres que não possuem o transtorno. Ju-Yu Yen et al (2020), verificaram que existe uma associação entre o TDPM e o Transtorno de Ansiedade 
Generalizada (TAG), sendo evidenciado que, uma mulher que já possua o TAG, tem até 7,65 vezes mais chances de desenvolver TDPM do que uma que não tem. Além disso, a associação dos dois transtornos se torna mais maléfica em relação à gravidade do TDPM com acentuação da ansiedade, depressão e irritabilidade durante a fase folicular. Śliwerski e BielawskaBatorowicz (2019) ainda afirmam que mulheres com a Síndrome Pré-Menstrual têm uma visão mais negativa de si mesmas, do mundo e do futuro, usando mais adjetivos negativos do que positivos para se descreverem.

Um outro estudo tailandês, no qual verificou-se a prevalência de SPM e seus impactos na vida de adolescentes, utilizando os critérios da ACOG (2009), Buddhabunyakan et al (2017) verificaram que esta síndrome foi associada à atividade educacional com uma maior falta de concentração e motivação, notas mais baixas e desempenho nas atividades comprometido, entretanto, os autores não observaram associação com absenteísmo e dificuldade no trabalho, além da qualidade das relações interpessoais. Divergindo dos autores supracitados, um estudo realizado especificamente sobre a SPM e suas consequências no trabalho mostrou que esse problema está associado à várias queixas ocupacionais, tais como dificuldade de concentração e realização de atividades, faltas durante o período menstrual e jornadas de trabalhos mais curtas devido aos sintomas (Hardy \& Hunter, 2021). Weise et al (2019) reafirmaram ainda que a SPM severa (TDPM) está associada ao sofrimento significativo, gerando interferência no dia a dia no trabalho bem como na família, no desempenho social e nos relacionamentos.

A vida sexual ativa mostrou-se influenciadora da SPM severa, corroborando com os resultados dos estudos de Abeje e Berhanu (2019), que dentre outras variáveis, associa a vida sexual ativa à SPM. No que se refere a orientação sexual, não foi observado influência na classificação da SPM e também não foram encontrados nas bases de dados pesquisadas outros artigos relacionando tal variável ao surgimento da SPM ou TDPM. Outras características foram relacionadas à síndrome, como consumo de cafeína, grandes quantidades de sal e de doces, comidas junk food, história familiar de SPM, além do sedentarismo, tarefas pesadas, menarca precoce e vida sexual ativa (Abeje \& Berhanu, 2019; Bhuvaneswari et al., 2019), etnia e cultura, status econômico, tabagismo e consumo de álcool (Buddhabunyakan et al., 2017), além de um sono irregular (Meers et al., 2019).

O estudo apresentou algumas limitações por se tratar de um estudo transversal, com informações retrospectivas, sujeito a problemas como viés de memória, uma vez que não mostra os dados reais da população, pois se coletarmos informações prospectivamente, poderia haver possibilidade de os resultados serem diferentes. Além disso, por ter sido feito em período de pandemia, contou com uma amostra bem reduzida quando comparada com a amostra inicial.

\section{Conclusão}

Os resultados encontrados neste estudo apontam uma alta prevalência de mulheres com síndrome pré-menstrual classificada como severa, além de apresentarem distúrbios físicos e emocionais decorrentes da SPM. Após realização da regressão, pôde-se perceber a influência da cor da pele, vida sexual, renda familiar, regularidade e duração menstrual na severidade da SPM. Entretanto, a faixa etária, grau de escolaridade e o tempo do ciclo menstrual não influenciaram na classificação da SPM.

Por fim, como sugestão a trabalhos futuros, seria interessante a realização de estudos longitudinais que possam mapear e analisar os reais fatores que influenciam no surgimento e na persistência da sintomatologia da SPM, acompanhando essas mulheres por pelo menos três ciclos menstruais, tempo recomendado pelo Colégio Americano de Ginecologia e Obstetrícia, bem como investigar medidas preventivas destas consequências e possíveis intervenções, de modo que em breve seja possível desenvolver um protocolo fisioterapêutico específico para a Síndrome Pré-Menstrual em todos os seus níveis. 


\section{Referências}

Abeje, A., \& Berhanu, Z. (2019). Premenstrual syndrome and factors associated with it among secondary and preparatory school students in Debremarkos town, North-west Ethiopia, 2016. BMC Research Notes, 12(1), 1-5. https://doi.org/10.1186/s13104-019-4549-9

Alves, M. H. F., Ribeiro, G. O., Vitorino, G. da S., Andrade, A. A. de, Uchôa, E. P. B. L., \& Carvalho, V. C. P. de. (2019). Prevalência da tensão prémenstrual entre universitárias. Fisioterapia Brasil, 20(3), 392-399. https://doi.org/https://doi.org/10.33233/fb.v20i3.2215

American College of Obstetricians and Gynecologists. (2009). Guidelines for Women's Health Care. A Resource Manual 3rd Edition. Journal of Obstetrics and Gynaecology, 29(1), 80-80. https://doi.org/10.1080/01443610802628957

American Psychiatric Association. (2014). DSM-5: Manual diagnóstico e estatístico de transtornos mentais (5th ed.). Artmed Editora.

Arruda, C., Fernandes, A., Cezarino, P., \& Simões, R. (2011). Tensão Pré-Menstrual. In Federação Brasileira das Associações de Ginecologia e Obstetrícia Sociedade Brasileira de Medicina de Família e Comunidade. Associação Médica Brasileira e Conselho Federal de Medicina.

Bhuvaneswari, K., Rabindran, P., \& Bharadwaj, B. (2019). Prevalence of premenstrual syndrome and its impact on quality of life among selected college students in Puducherry. National Medical Journal of India, 32(1), 17-19. https://doi.org/10.4103/0970-258X.272109

Buddhabunyakan, N., Kaewrudee, S., Chongsomchai, C., Soontrapa, S., Somboonporn, W., \& Sothornwit, J. (2017). Premenstrual syndrome (PMS) among high school students. Int J Womens Health, 21(9), 501-505. https://doi.org/10.2147/IJWH.S140679

CNS - Conselho Nacional de Saúde. (2012). Resolução $N^{o}$ 466, De 12 De Dezembro De 2012. Diário Oficial Da União. https://bvsms.saude.gov.br/bvs/saudelegis/cns/2013/res0466_12_12_2012.html

Geleski, A. C., Benincá, S. C., Vieira, D. G., Freitas, S. P. de, \& Mazur, C. E. (2018). Sintomas envolvidos, consumo alimentar e estado nutricional de universitárias durante o período menstrual. Revista de Atenção à Saúde, 16(56), 5-11. https://doi.org/https://doi.org/10.13037/ras.vol16n56.4591

Hardy, C., \& Hunter, M. S. (2021). Premenstrual symptoms and work: Exploring female staff experiences and recommendations for workplaces. International Journal of Environmental Research and Public Health, 18(7). https://doi.org/10.3390/ijerph18073647

Hochman, B., Nahas, X. F., Filho, R. S. de O., \& Ferreira, L. M. (2005). Desenhos de pesquisa. Acta Cirúrgica Brasileira, 20(2), 1-8.

Jang, D., \& Elfenbein, H. A. (2019). Menstrual Cycle Effects on Mental Health Outcomes: A Meta-Analysis. Archives of Suicide Research, 23(2), 312-332. https://doi.org/10.1080/13811118.2018.1430638

Jarosz, A. C., Jamnik, J., \& El-Sohemy, A. (2017). Hormonal contraceptive use and prevalence of premenstrual symptoms in a multiethnic Canadian population. BMC Women's Health, 26(17(1)), 87. https://doi.org/10.1186/s12905-017-0450-7.

Macambira, M. O., \& Golino, H. (2018). Evidências de validade da escala de síndrome pré-menstrual. Avaliação Psicológica, 17(2), 180-187. https://doi.org/http://dx.doi.org/10.15689/ap.2018.1702.13662.03

Maia, M. da S., Aguiar, M. I. F. de, Chaves, E. S., \& Rolim, I. L. T. P. (2014). Qualidade de vida de mulheres com tensão pré-menstrual a partir da escala WHOQOL-BREF. Ciência, Cuidado E Saúde, 13(2), 236-244. https://doi.org/https://doi.org/10.4025/cienccuidsaude.v13i2.15759

Meers, J. M., Bower, J. L., \& Alfano, C. A. (2019). Poor sleep and emotion dysregulation mediate the associationbetween depressive and premenstrual symptoms in youngadult women. Archives of Women's Mental Health, 23, 351-359. https://doi.org/https://doi.org/10.1007/s00737-019-00984-2

Muramatsu, C. H., Vieira, O. C. S., Simões, C. C., Katayama, D. A., \& Nakagawa, F. H. (2001, September). Consequências da síndrome da tensão prémenstrual na vida da mulher. Revista Da Escola de Enfermagem Da USP, 35(3), 205-213. https://doi.org/10.1590/S0080-62342001000300002

Paiva, S. de P. C., Paula, L. B., \& Nascimento, L. L. O. do. (2010). Tensão pré-menstrual (TPM): uma revisão baseada em evidências científicas. Femina, $38(6), 311-315$.

Pereira, A. S., Shitsuka, D. M., Parreira, F. J., \& Shitsuka, R. (2018). Metodologia da pesquisa científica. In Repositório digital da UFSM. 119.

Santos, C. C. Dos, Alberton, O., Belettini, S. T., Donadel, G., Dalmagro, M., Otenio, J. K., Pinc, M. M., Sabec, G. Z., \& Lourenço, E. L. B. (2020). Tratamento alternativo da síndrome pré-menstrual com camomila, maçã e maracujá. Research, Society and Development, 9(10), e2929108702. https://doi.org/https://doi.org/10.33448/rsd-v9i10.8702

Schoep, M. E., Nieboer, T. E., van der Zanden, M., Braat, D. D. M., \& Nap, A. W. (2019). The impact of menstrual symptoms on everyday life: a survey among 42,879 women. American Journal of Obstetrics and Gynecology, 220(6), 569.e1-569.e7. https://doi.org/10.1016/j.ajog.2019.02.048

Shrestha, D. B., Shrestha, S., Dangol, D., Aryal, B. B., Shrestha, S., Sapkota, B., \& Rai, S. (2019). Premenstrual Syndrome in Students of a Teaching Hospital. J Nepal Health Res Counc, 17(43), 253-257. https://doi.org/https://doi.org/10.33314/jnhrc.v0i0.1213

Silva, E. D. F. da, Giacon, F. P., \& Gonçalves, J. A. da S. (2020). Efeito da drenagem linfática na síndrome da tensão pré menstrual. Brazilian Journal of Health Review, 3(5), 14072-14094. https://doi.org/10.34119/bjhrv3n5-215

Śliwerski, A., \& Bielawska-Batorowicz, E. (2019). Negative cognitive styles as risk factors for the occurrence of PMS and PMDD. Journal of Reproductive and Infant Psychology, 37(3), 322-337. https://doi.org/10.1080/02646838.2018.1543943

Tolossa, F. W., \& Bekele, M. L. (2014). Prevalence, impacts and medical managements of premenstrual syndrome among female students: Cross-sectional study in college of health sciences, Mekelle University, Mekelle, Northern Ethiopia. BMC Women's Health, 14(1), 1-9. https://doi.org/10.1186/1472-6874-1452 
Research, Society and Development, v. 10, n. 8, e6510817116, 2021

(CC BY 4.0) | ISSN 2525-3409 | DOI: http://dx.doi.org/10.33448/rsd-v10i8.17116

Victor, F. F., Souza, A. I., Barreiros, C. D. T., Barros, J. L. N. De, Silva, F. A. C. Da, \& Ferreira, A. L. C. G. (2019). Quality of Life among University Students with Premenstrual Syndrome. Revista Brasileira de Ginecologia e Obstetricia, 41(5), 312-317. https://doi.org/10.1055/s-0039-1688709

Weise, C., Gudrun, K., \& Maria, K. (2019). Reply to 'Questioning the Beneficial Effects of Internet-Based Cognitive Behavioral Therapy on Premenstrual Dysphoric Disorder. Gale Academic OneFile, 88(4), 237.

Yen, J. Y., Lin, P. C., Huang, M. F., Chou, W. P., Long, C. Y., \& Ko, C. H. (2020). Association between generalized anxiety disorder and premenstrual dysphoric disorder in a diagnostic interviewing study. International Journal of Environmental Research and Public Health, 17(3). https://doi.org/10.3390/ijerph17030988

Yilmaz-Akyuz, E., \& Aydin-Kartal, Y. (2019). O efeito da dieta e do exercício aeróbio na Síndrome Pré-menstrual: ensaio controlado de modo aleatório. Rev. Nutr., 1-10. https://doi.org/http://dx.doi.org/10.1590/1678-9865201932e180246 